\section{Abklärung von postmenopausalen Blutungen (PMPB)}

\author{
L. Bronz, E. Dreher, A. Almendral, A. Studer, U. Haller
}

Arbeitsgruppe PMPB der Kommission Qualitätssicherung der Schweizerischen Gesellschaft für Gynäkologie und Geburtshilfe

\section{Präambel}

Aufbauend auf Evidence-based Medicine (EBM) sollen Leitlinien eine Hilfeleistung und Orientierung im Sinne einer Effizienzsteigerung von Diagnostik und Therapie an der individuellen Patientin und im Sinne der Vertrauensbildung in die ärztliche Entscheidung erbringen [1-3]. "Leitlinien" sind systematisch entwickelte Darstellungen und Empfehlungen mit dem Zweck, Arzt und Patientin bei der Entscheidung über zweckdienliche Massnahmen der Krankenversorgung (Prävention, Diagnostik, Therapie und Nachbehandlung) unter spezifischen klinischen Umständen $\mathrm{zu}$ unterstützen [4]. Leitlinien stellen den Stand des Wissens über effektive und zweckdienliche Krankenversorgung zum Zeitpunkt der Veröffentlichung dar. Wegen der Fortschritte der wissenschaftlichen Erkenntnisse müssen Leitlinien periodisch überarbeitet werden. Die Entscheidung darüber, ob einer bestimmten Empfehlung gefolgt werden soll, muss vom Arzt unter Berücksichtigung der bei der individuellen Patientin vorliegenden Gegebenheiten und der verfügbaren Ressourcen entschieden werden [5, 6]. Im Einzelfall müssen somit die aktuelle Situation der Patientin, ihr Umfeld, ihre sozio-ökonomische Situation, die Komorbidität und Nebendiagnosen, aber

Korrespondenz:

Dr. med. Lucio Bronz

Ospedale Regionale Bellinzona e Valli

CH-6500 Bellinzona auch ihre ethische und religiöse Haltung mitberücksichtigt werden. Damit ist ein entscheidender ärztlicher Freiraum gegeben, welcher es überhaupt ermöglicht, Leitlinien in die Praxis umzusetzen. «Therapeutische Freiheit” bedeutet, dass der Arzt sowohl die Kompetenz hat wie auch die Verantwortung dafür trägt, dass die von ihm an der Patientin vorgenommene Diagnostik bzw. durchgeführte Therapie dem aktuellen Stand der Wissenschaft entspricht - therapeutische Freiheit somit gleichermassen als Freiheit wie auch als Verpflichtung, das Richtige zu tun [7].

Das Erstellen und die laufende Aktualisierung von Leitlinien erfordern hohe Kompetenz, Beharrungsvermögen und zeitlichen Einsatz. Vieles im Medizinberuf basierte schon immer auf Evidenz, neu sind eher die Methoden, Fortschritte und Kriterien, die eine Beurteilung der Validität von Studien und eine verständliche Darstellung ihrer Resultate ermöglichen. Eine gute Leitlinie zeigt unter anderem auf, für welche Situationen sie Handlungsempfehlungen anbietet, wie aktuell die Handlungsanweisung ist, welche Grade der wissenschaftlichen Sicherung für jede einzelne Aussage besteht und inwiefern ihre Befolgung der Patientin voraussichtlich Nutzen bringen wird [7]. Gute Leitlinien werden den Diskurs zwischen Medizin und Recht verbessern («decision making processes").

\section{Literatur}

1 Haller U, Reinold E, Hepp H. "Evidence Based Medicine» Leitlinien als Bedrohung oder Notwendigkeit für Arzt und Patient? Gynäkol Geburtshilfliche Rundsch 1998;38:1-2.

2 Zentralvorstand der FMH. Guideline für Guidelines: Schweiz Ärztezeitung 1999;80:581-3.

3 Steurer J. Kritische Beurteilung von «Guidelines». Praxis 1998;87:199-204.

4 AWMF: Definition und Erläuterung des Begriffs «Leitlinie»: Orientierung an der Definition der "Agency for Health Care Policy and Research" für die "Clinical Practice Guidelines» der USA. AWMF online: www.uniduesseldorf.de/WWW/AWMF/II/II_metho.htm

5 Fletcher RH. Clinical practice guidelines and the individual patient: Practice guidelines and the practice of medicine: Is it the end of clinical judgement and expertise? Schweiz Med Wochenschr 1998;128:1883-8.

6 Dans AL, Dans LF, Guyatt GH, Richardson S for the Evidencebased Medicine Working Group. Users guides to the medical literature: XIV. How to decide on the applicability of clinical trial results to your patient. JAMA 1998;279:545-7.

7 Kuhn HP. Keine Angst vor Guidelines: Schweiz Ärztezeitung 1999;80:583-6. 


\section{Einführung}

\subsection{Relevanz des Themas}

Etwa 5\% aller gynäkologischen Untersuchungen werden wegen PMPB durchgeführt [1]. Fast 70\% aller gynäkologischen Konsultationen bei peri- und postmenopausalen Frauen stehen im Zusammenhang mit Blutungsstörungen [2]. Die PMPB ist das wichtigste klinische Warnsignal für das Endometriumkarzinom [3] und ist bei etwa 80\% der Patientinnen mit einem Endometriumkarzinom das erste Symptom $[4,5]$.

In über $60 \%$ der Fälle von postmenopausaler Blutung findet sich keine organische Ursache oder eine Atrophie des Endometriums. Da aber in 10-15\% ein Endometriumkarzinom die Ursache ist, muss eine Abklärung erfolgen.

Gründe für eine uterine postmenopausale Blutung sind [6-13]: Endometriumatrophie (60-80\%), Östrogenersatztherapie (15-25\%), Endometriumpolypen (2-12\%), Endometriumhyperplasien (5-10\%), Endometriumkarzinome (10-15\%).

Es handelt sich bei der PMPB um ein Problem, dem man in der Praxis häufig begegnet, und das somit von grosser klinischer Bedeutung ist, jedoch sowohl diagnostisch als auch therapeutisch recht unterschiedlich angegangen wird.

\subsection{Ziel der Empfehlungen}

Die Empfehlungen sollen der Ärztin/dem Arzt den Stand der wissenschaftlichen Evidenz für die Vorgehensweise zur Abklärung von postmenopausalen Blutungen aufzeigen mit dem Ziel

- ein Endometriumkarzinom und seine Vorstufen frühzeitig zu erfassen;

- gutartige postmenopausale Blutungsursachen zu erkennen;

- invasive Eingriffe zu minimieren.

\subsection{Methode}

Um einen schweizerischen Konsens $\mathrm{zu}$ erreichen, wurde für die vorliegende Leitlinie gemäss Vorgaben der Kommission Qualitätssicherung der SGGG wie folgt vorgegangen:

- Die Kommission Qualitätssicherung beauftragte eine Arbeitsgruppe unter der Leitung von Dr. L. Bronz, Bellinzona, mit der Ausarbeitung der Leitlinie.

- Die vier Experten dieser Arbeitsgruppe trafen sich während zwei Jahren mehrmals, um die Ergebnisse, welche zwischen den Sitzungen in Homerating-Runden erarbeitet wurden, abzustimmen. Es erfolgten acht Revisionen. Eine Überarbeitung ist in zwei Jahren vorgesehen.

- Die Erstellung dieser Leitlinie erfolgte strikte innerhalb der SGGG und wurde in keiner Weise gesponsert; es besteht keinerlei Abhängigkeit.

- Die Literatursuche wurde mit Hilfe der MedlineDatenbank für den Zeitraum von 1990 bis Juli 1998 unter folgenden Stichworten durchgeführt: Postmenopausenblutung, Sonographie, Hysteroskopie, Dilatation und Curettage. Dazu wurde die ganze zur Verfügung stehende deutschsprachige und englische Literatur systematisch analysiert.

- Im Rahmen ihrer regelmässigen Sitzungen überprüfte die Kommission Qualitätssicherung die jeweiligen Vorschläge und erteilte Überarbeitungsaufträge.

- Nach Verabschiedung durch die Kommission Qualitätssicherung wurde die Leitlinie während zwei Monaten von 60 Mitgliedern der SGGG im klinischen Test überprüft und schliesslich unter Berücksichtigung dieser Erfahrungswerte dem Vorstand der SGGG unterbreitet.

- Die Implementierung der Leitlinien erfolgt über Publikationen in den Printmedien, Verbreitung durch Internet, Aufnahme durch die Akademie für Fortbildung der Schweizerischen Gesellschaft für Gynäkologie und Geburtshilfe (SGGG) und Einsatz in den Weiter- und Fortbildungsveranstaltungen der SGGG. Jedes Mitglied der SGGG erhält ein Exemplar zugestellt.

- Zu den einzelnen klinisch entscheidenden Aussagen der Leitlinie wurde der Grad der wissenschaftlichen Sicherung gemäss der Klassifikation der "Canadian Task Force on the Periodic Health Examination" beurteilt und angegeben [14]. Damit lässt sich aussagen, ob die einzelnen Entscheidungen hauptsächlich auf Evidenz oder Konsens zustande gekommen sind.

Grad der Evidenz ("Canadian Task Force on the Periodic Health Examination" [14]):

- Grad I: Die Evidenz ist aufgrund randomisierter kontrollierter Studien (oder Metaanalysen) von genügendem Umfang derart, dass die Gefahr, dass sie falsch positive oder falsch negative Resultate beinhalten, gering ist.

- Grad II: Die Evidenz basiert auf randomisierten kontrollierten Studien, welche jedoch zu klein sind, um ihnen Grad I zuzusprechen; sie können positive Trends, welche jedoch statistisch nicht signifikant sind, oder gar keine Trends zeigen. Sie sind mit einem hohen Risiko falsch negativer Resultate verbunden.

- Grad III: Die Evidenz basiert auf nicht randomisierten Kontroll- oder Kohortenstudien, Fallserien, Fallkontrollstudien oder Querschnittstudien.

- Grad IV: Die Evidenz basiert auf der Meinung angesehener Experten oder Expertengremien, wie sie in publizierten Konsenskonferenzen oder in Guidelines angegeben werden.

- Grad V: Die Evidenz basiert auf der Meinung derjenigen Personen, welche diese Guidelines geschrieben oder aktualisiert haben, beruhend auf ihrer Erfahrung, ihrer Kenntnis der einschlägigen Literatur und der Diskussion mit ihren Fachkollegen.

\subsection{Definition und Zielgruppe}

Bezieht sich auf Frauen, die frühestens zwölf Monate nach der letzten Menstruation eine genitale Blutung aufweisen. 


\section{Zusammenfassung der Empfehlungen}

2.1 Die Anamnese und die klinisch-gynäkologische Untersuchung mit Entnahme eines PAP-Abstriches bilden den ersten Schritt der Abklärung

- Die Anamnese soll sicherstellen, ob es sich wirklich um eine Blutung aus dem genitalen und nicht aus dem urologischen oder intestinalen Bereich handelt. Die Einnahme von Medikamenten ist festzuhalten und auf Risikofaktoren des Endometriumkarzinoms muss geachtet werden. Die weitere Abklärung wird dadurch nicht beeinflusst.

- Die klinisch-gynäkologische Untersuchung hat die Quelle einer PMPB nach anatomischer Lokalisation - uterin, infra- oder suprauterin - nachzuweisen. Infrauterine Blutungsursachen sind mit der Inspektion des äusseren Genitale und mit der Spekulumuntersuchung leicht zu diagnostizieren. Uterine Ursachen stehen weit im Vordergrund.

- Die Zytologie und die Kolposkopie, ergänzt durch die bimanuelle Untersuchung, lassen ein Zervixkarzinom als Ursache ausschliessen. Im zytologischen Abstrich erkennbare atypische endometriale Zellen wecken den Verdacht auf ein Endometriumkarzinom.

2.2 Die transvaginale Sonographie (TVS) bildet den nächsten Schritt, falls obige Abklärungen negativ ausgefallen sind

- Immer sollen beide Adnexe inspiziert und sonographisch dokumentiert werden. Zystisch-solide Massen im Adnexbereich lassen sich damit als suprauterine Ursache einer PMPB besser diagnostizieren.

- Danach soll der Uterus kontrolliert werden. Die Messung des Endometriums im Longitudinalschnitt auf Höhe der maximalen Endometriumdicke entscheidet über das weitere Vorgehen.

- Falls die Endometriumdicke $\leq 4 \mathrm{~mm}$ misst, kann vorerst abgewartet werden. Die Patientin soll nach drei Monaten mit TVS wieder kontrolliert werden. Falls sie danach wieder blutet oder die Endometriumdicke bei TVS $>4 \mathrm{~mm}$ misst, ist gemäss Ziffer 2.3 vorzugehen.

- Falls die Endometriumdicke $>4 \mathrm{~mm}$ misst oder falls sie nicht messbar ist, soll eine histomorphologische Abklärung gemäss Ziffer 2.3 durchgeführt werden.

- Die Hydrosonographie eignet sich zuerst in diesen Fällen als einfache Methode zur Ergänzung der TVS. Sie kann beim Entscheid mithelfen, welche weiteren invasiveren Abklärungen durchgeführt werden sollen (Endometriumbiopsie/hysteroskopische Resektion).

- Computertomographie (CT) oder Magnet-Resonanz-Tomographie (MRT) sind in der Regel bei der Abklärung von Patientinnen mit PMPB nicht indiziert.
2.3 Nur die histologische Untersuchung des entnommenen Materials ermöglicht eine definitive Diagnose

- Ergeben die TVS oder die Hydrosonographie einen polypösen Befund, ist die hysteroskopisch gezielte Entfernung die diagnostische Methode der Wahl.

- Bei einer symmetrischen oder asymmetrischen Endometriumverdickung bei der Hydrosonographie kann die weniger invasive Endometriumbiopsie genügen. Falls mit dieser Methode aber kein repräsentatives Material vorliegt, soll wie oben vorgegangen werden.

- Eine fraktionierte Curettage sollte in der Regel nicht mehr allein durchgeführt, sondern mit einer Hysteroskopie kombiniert werden.

\section{Begründungen zu den Empfehlungen}

ad 2.1 Die Anamnese und die klinisch-gynäkologische Untersuchung mit Entnahme eines PAP-Abstriches bilden den ersten Schritt zur Untersuchung

Die Anamnese ergibt Hinweise auf eine unregelmässig durchgeführte Hormonersatzbehandlung oder eine allfällige neu begonnene Therapie mit gerinnungshemmenden Medikamenten. In den meisten Fällen einer PMPB liegt keine organische Ursache vor. Trotzdem ist es wichtig, jede maligne und benigne organische Ursache auszuschliessen.

Der Anamnese folgt die klinisch-gynäkologische Untersuchung. Falls nötig, werden weitere diagnostische Massnahmen getroffen (transvaginale Sonographie, Hydrosonographie), bevor die definitive histologische Diagnose durch invasivere Methoden erzwungen wird (Hysteroskopie, fraktionierte Curettage, Hysterektomie).

Das Ziel geht dahin, durch ein Minimum an nichtinvasiven und invasiven Eingriffen die Ursache der PMPB aufzudecken, um eine allfällig nötige Therapie gezielt einzuleiten.

- Die Anamnese soll festhalten, ob es sich wirklich um eine PMPB handelt und nicht um eine Blutung aus dem urologischen oder intestinalen Bereich. Die Einnahme von Medikamenten (Hormone, Tamoxifen, usw.) ist zu protokollieren und auf Risikofaktoren des Endometriumkarzinoms (Hypertonie, Diabetes mellitus, St. n. Mammakarzinom) soll geachtet werden.

Die Menopause ist definiert als der Zeitpunkt der letzten, ovariell gesteuerten Menstruation und liegt heute im Mittel bei 51 Jahren. Die Postmenopause (PMP) ist der Zeitraum zwischen Menopause und Senium; sie beginnt zwölf Monate nach der letzten spontanen Menstruation [15].

Man spricht von PMPB, wenn nach einem Intervall von mehr als 12 Monaten nach der letzten Menstruation wieder eine Blutung auftritt.

Diese Definition gilt auch für jede Patientin, die unter Medikamenteneinnahme steht (Hormonsubstitutionstherapie, Tamoxifen, andere). 
Sehr selten wird über Blasenkarzinome $[5,16,17]$ oder Kolonkarzinome [16] als Ursache einer PMPB berichtet; deshalb sollte bei der Anamnese sorgfältig der genitale Ursprung der Blutung erfragt werden.

Da das Endometriumkarzinom die häufigste maligne Ursache einer PMPB ist, sollte nach seinen Risikofaktoren wie Diabetes mellitus, Hypertonie, Adipositas und St. n. Mammakarzinom, Einnahme von Östrogenen allein ohne Gestagenzusatz, Tamoxifen gefragt werden. Der Abklärungsvorgang einer Patientin mit PMPB unter Tamoxifen ist im Prinzip identisch wie bei einer Patientin mit PMPB ohne Tamoxifen [18].

- Die klinisch-gynäkologische Untersuchung dient dazu, die Quelle einer PMPB nach anatomischer Lokalisation - uterin, infra- oder suprauterin festzustellen.

Infrauterine Blutungsursachen (Vulva, Vagina) sind mit der Inspektion des äusseren Genitale und mit der Spekulumuntersuchung leicht zu diagnostizieren.

Suprauterine Blutungsursachen (Tuben, Ovarien) sind durch die Palpation der inneren Genitalorgane schwieriger zu erfassen, werden aber häufig durch die transvaginale Sonographie entdeckt $[5,13,16,19$, 20], wobei man an erster Stelle gutartige Ovarialzysten findet, gefolgt von bösartigen Ovarialtumoren.

Die uterinen sind die häufigsten Ursachen einer PMPB.

Die Häufigkeit des Zervixkarzinoms wird sehr unterschiedlich angegeben, zwischen 0,8-12,9\% der Patientinnen mit einer PMPB [7, 11, 13, 19]. Entsprechend stark variiert in der Literatur das Verhältnis Korpuskarzinom: Zervixkarzinom zwischen 1/1 [7] bis $\mathrm{zu} 10 / 1$ [11].

Das Zervixkarzinom kann mit der gynäkologischen Untersuchung (Spekulum, Kolposkopie), ergänzt durch die Zytologie oder Biopsie, mit höchster Treffsicherheit diagnostiziert werden. Diese Abklärungsschritte müssen immer an erster Stelle stehen. Erweist sich der Uterus bei der bimanuellen Untersuchung als vergrössert, ist an submuköse Myome [21, 22, 23, 24] oder seltener an Sarkome [1, 10, 17] als Blutungsursachen zu denken.

Ist der Uterus palpatorisch nicht vergrössert, ist eine organische korporale Ursache aber nicht ausgeschlossen. Die Effizienz der klinischen Untersuchung ist von der Erfahrung des Untersuchers abhängig. Sie spielt aber nur eine untergeordnete Rolle bei der Diagnose der korporalen postmenopausalen Blutungsursachen.

ad 2.2 Die nicht invasive Methode der Wahl zur weiteren Abklärung einer PMPB ist die transvaginale Sonographie (TVS)

Gutartige Ovarialzysten und Ovarialkarzinome können eine PMPB verursachen. Häufig werden sie bei der gynäkologischen Untersuchung gar nicht entdeckt [19].

Die TVS kann zystische und solide Massen im Adnexbereich erfassen und teilweise auch differenzieren [5, 13, 16, 19, 20] (Evidenz III). Bei jeder Ultra- schalluntersuchung sollen beide Adnexe inspiziert und dokumentiert werden.

Bei den korporalen Ursachen findet man in der bisher grössten, multizentrisch, prospektiv durchgeführten und 1995 publizierten Studie [11], gemäss Histologie, der Häufigkeit nach: atrophes Endometrium (59\%), benigne Polypen (12,4\%), Endometriumkarzinom (10,1\%), Hyperplasien des Endometriums (9,9\%), noch aktives Endometrium (6,8\%), andere seltenere Ursachen (1,8\%) (Evidenz III).

In dieser Studie wird nicht differenziert, ob bei der Hyperplasie eine Atypie vorhanden ist oder nicht. Ihr prozentualer Anteil in der Literatur variiert stark $[3,4,9,19,24,25]$ von $1,2 \%$ bis $13 \%$.

Weiterhin ist die Inzidenz von Myomen nicht erwähnt, da die histologische Diagnose hauptsächlich an durch fraktionierte Curettage gewonnenem Gewebematerial erstellt wurde.

Submuköse Myome findet man bei Patientinnen mit PMPB in 6-7\% [22, 24], ihr prozentualer Anteil ist massiv erhöht bei den Autoren, die die Ursachen einer PMPB mittels operativer Hysteroskopie behoben haben: $35-48 \%[21,23]$.

Obwohl nur die histologische Untersuchung des entnommenen Materials eine definitive Diagnose erlaubt, muss man im Auge behalten, dass in rund 50-60\% der Fälle die Histologie ein atrophes Endometrium ergibt; in folgenden Fällen ist eine histologische Untersuchung prinzipiell unnötig:

- Die TVS ist eine geeignete Methode zur Beurteilung des Endometriums. Bei einer Endometriumdicke $\leq 4 \mathrm{~mm}$ kann auf eine histologische Abklärung verzichtet werden.

Die Messung des Endometriums erfolgt im Longitudinalschnitt auf Höhe der maximalen Endometriumdicke (aussen-aussen). Bei flüssigkeitsgefülltem Cavum uteri wird der Flüssigkeitssaum gesondert vermessen und von der Gesamtdicke abgezogen [26, 27, 28]. Aus den Arbeiten, die detaillierte Angaben bezüglich Endometriumdicke und -histologie für verschiedene "cut-off levels" aufweisen, wurden mit dem "cut-off level" $\leq 4 \mathrm{~mm}$ und $>4 \mathrm{~mm}$ die entsprechend korrekten und nicht korrekten Ultraschalldiagnosen für die wichtigsten histologischen Diagnosen zusammengestellt (Tab. 1-4):

\section{Atrophie des Endometriums (Tab. 1)}

In 17 meist prospektiven Arbeiten bei insgesamt 4858 Patientinnen mit PMPB wurde in 2338 Fällen eine Atrophie diagnostiziert $(48,1 \%)$.

In 1822 von 2338 Fällen (77,9\%) war bei einem Cut-off von $\leq 4 \mathrm{~mm}$ Endometriumdicke die richtige Diagnose mittels TVS vermutet worden.

Bei weiteren 516 Frauen $(22,1 \%)$ mit der histologischen Diagnose Atrophie betrug die mittels TVS gemessene Endometriumdicke aber mehr als $4 \mathrm{~mm}$. Hier ist durch den Einsatz weiterer nicht-invasiver Abklärungen, zum Beispiel Hydrosonographie, eine Verbesserung der Diagnostik noch möglich.

Durch den Einsatz der TVS könnte man also allein mittels Bestimmung der Dicke des Endometriums 
in rund 50\% der Fälle (12-83,7\%) auf eine histomorphologische Abklärung verzichten mit entsprechender Kostensenkung [4, 5, 9, 10, 16, 20, 32, 33, 35, 36] (Evidenz III). Allerdings wurde in einer Arbeit darauf hingewiesen, dass das Alter und der Body Mass Index unabhängige Faktoren sind, die die Sensitivität und den positiven prädiktiven Wert bei einem Cut-off von $\leq 4 \mathrm{~mm}$ beeinflussen (z.B. bei einer 60jährigen Patientin steigt die Wahrscheinlichkeit einer Atrophie von 45\% auf 64\%, wenn der Body Mass Index von 35 auf 20 abnimmt) [20] (Evidenz III).

\section{Endometriumkarzinom (Tab. 2)}

In 24 meist prospektiven Arbeiten wurde bei insgesamt 5897 Patienten mit PMPB in 597 Fällen (10,1\%) ein Endometriumkarzinom histologisch verifiziert. 24 von 597 Frauen (4\%) wiesen bei der TVS eine Endometriumdicke von $\leq 4 \mathrm{~mm}$ auf. 13 dieser 24 Fälle entstammen einer einzigen Arbeit [8] (Evidenz III). Dabei wurde in 4 dieser 13 Fälle eine Endometriumdicke von 0 (also nicht messbar) protokolliert. Wenn man diese 24 Fälle auf die Anzahl Patientinnen mit PMPB bezieht, würde man in 0,4\% der Fälle (24/5897) mittels TVS ein Endometriumkarzinom verpassen.

In einer früheren Analyse, bei der ein Endometriumkarzinom in 11\% der Patientinnen mit PMPB diagnostiziert vorkam, fand man bei einer Endometriumdicke von $\leq 4 \mathrm{~mm}$ ein Endometriumkarzinom in 1,4\%. Wichtig ist, den mit 98,6\% sehr hohen negativen prädiktiven Wert dieser Messmethode hervorzuheben [10] (Evidenz III).

Endometriumpolypen (Tab. 3)

In 15 ebenfalls meist prospektiven Arbeiten bei insgesamt 4404 Patientinnen mit PMPB wurden in 492 Fällen (11,2\%) Endometriumpolypen diagnostiziert.

Bei 55 Fällen von Polypen war die Endometriumdicke $\leq 4 \mathrm{~mm}(11,2 \%)$. Somit hätte man mit alleiniger Messung der Endometriumdicke mittels TVS in 1,2\% (55/4404) die Diagnose verpasst.

Hyperplasie des Endometriums (Tab. 4)

In 17 wiederum meist prospektiven Arbeiten fand man bei 4732 Patientinnen mit PMPB in 599 Fällen $(12,3 \%)$ eine histologisch gesicherte Endometriumhyperplasie. $49 \mathrm{mal}$, d.h. in 8,2\% betrug die Endometriumdicke $<4 \mathrm{~mm}$, so dass die Hyperplasie nicht diagnostiziert wurde. Mit der TVS verpasste man also in 1\% der Fälle (49 auf 4732) die korrekte Diagnose.

Diese Analyse lässt den Schluss zu, dass mit der alleinigen Messung der Endometriumdicke bei einem Cut-off von $5 \mathrm{~mm}$ in 2,6\% der Fälle pathologische Endometriumbefunde nicht erfasst werden. Für die Polypen macht der Anteil 1,2\%, für die Hyperplasie $1 \%$ und für das Endometriumkarzinom 0,4\% aus.

Im Nordic-Trial [11] erwies sich der Anteil verpasster Befunde mit 5,5\% als doppelt so hoch. Obwohl international kein Konsens über den Cut-off vorliegt, scheint die Limite von $5 \mathrm{~mm}$ eine vernünftige Lösung zu sein. Darunter darf vorerst auf eine histomorphologische Diagnose verzichtet werden. Nachfolgende Kontrollen sind aber notwendig [10, 41] (Evidenz IV).
- Falls das Endometrium bei TVS nicht messbar ist oder falls die Endometriumdicke $>4 \mathrm{~mm}$ beträgt, müssen weitere Abklärungen durchgeführt werden.

Im Nordic-Trial konnte bei 30 Patientinnen $(2,8 \%)$ das Endometrium mit der TVS nicht gemessen werden, darunter fand sich ein Fall mit einem Endometriumkarzinom [11] (Evidenz III). In einer weiteren Studie erwies sich das Endometrium unter 29 Patientinnen mit Endometriumkarzinom in 4 Fällen als nicht messbar [8] (Evidenz III).

Aus der obigen Analyse geht hervor, dass die meisten benignen und malignen Veränderungen des Endometriums eine Endometriumdicke von $>4 \mathrm{~mm}$ zeigen. Darunter befinden sich Polypen, Hyperplasien, atypische Hyperplasien, Endometriumkarzinome, aber auch 22,1\% der Atrophien.

Die Messung der Endometriumdicke mit der TVS ist also sehr sensitiv, aber auch wenig spezifisch. Um die Spezifizität zu erhöhen, haben gewisse Autoren zusätzliche Kriterien zur Beurteilung des Endometriums eingeführt: die Echogenität und die Homogenität [27, 42]. Bezüglich der Reproduzierbarkeit der Messungen sei der Hinweis auf eine Arbeit gegeben, die zeigt, dass Unerfahrene stark variierende Messresultate erhalten, d.h., dass zur Minimierung der Fehlerquote grosse Übung nötig ist [43] (Evidenz III).

Aus den meisten Arbeiten geht hervor, dass bei einer Endometriumdicke $\leq 4 \mathrm{~mm}$ kaum pathologische Veränderungen zu erwarten sind, wohl aber fast sicher bei Messresultaten >10 mm. Die Grauzone, die bis mehrals 1/3 der pathologischen Alterationen umfasst, liegt bei einer Endometriumdicke von 5-10 mm [43].

- Die Hydrosonographie eignet sich als einfache Methode zur Ergänzung der TVS bei einer Endometriumdicke von $>4 \mathrm{~mm}$.

Ihre Durchführung ist leicht, sie wird von der Patientin gut ertragen und gibt bessere Auskünfte über die pathologischen Veränderungen im Cavum uteri. Die Hydrosonographie erleichtert ferner den Entscheid, welche weiteren invasiven Abklärungen durchgeführt werden sollen. Somit könnte man auch auf weitere 50\% unnötiger Eingriffe verzichten. Dieses Verfahren ist spezifischer als die alleinige Messung der Endometriumdicke, indem intrakavitäre Prozesse (Polypen/Myome) und asymmetrische Endometriumverdickungen besser dargestellt werden. Ihr Wert wird mit dem der diagnostischen Hysteroskopie verglichen. In 3-8\% der Fälle ist die Hydrosonographie, meist wegen einer Zervikalkanalstenose, nicht durchführbar [22, 24, 44-48] (Evidenz III).

Ergibt die Hydrosonographie den Verdacht auf einen Polypen oder ein submuköses Myom, wird der nächste Schritt direkt die operative Hysteroskopie mit Resektion des Befundes (Schlinge oder Laser) sein [21, 23]. Falls eine asymmetrische Endometriumverdickung oder der Verdacht auf ein Endometriumkarzinom vorliegt (unregelmässige Begrenzung, Infiltration des Myometriums), ist eine hysteroskopisch gezielte Endometriumbiopsie die Abklärung der Wahl [19]. 
Folgende Tatbestände fordern bei der postmenopausalen Blutung neben der Vaginalsonographie eine weitere Abklärung: die rezidivierende PMPB, Endometrium sonographisch $>4 \mathrm{~mm}$ oder nicht messbar, endometrale Zellen im PAP-Abstrich, ungenügendes, atypisches oder zervikales Gewebematerial bei einer diagnostischen Biopsie (Pipelle oder Vabra) [49, 50].

ad 2.3 Die morphologische Abklärung: Nur die histologische Untersuchung des entnommenen Materials ermöglicht eine definitive Diagnose. Die Abklärung kann in Stufen zunehmender Invasivität erfolgen

- Endometriumbiopsien, zum Beispiel Pipelle, Vabra, erreichen eine hohe Treffsicherheit bei der Diagnose des Endometriumkarzinoms, sind jedoch zur Erfassung gutartiger Veränderungen (z.B. Polypen) ungenügend.

Die Pipelle de Cornier oder Vabra Aspirationscurettage haben, insbesondere in Kombination mit einer Vaginalsonographie, eine Sensitivität von bis zu $100 \%$ in der Entdeckung des Endometriumkarzinoms, sind aber weniger gut geeignet für den Nachweis benigner uteriner Blutungsursachen (submuköse Myome, Polypen), welche sich bei PMPB in bis $\mathrm{zu}$ 90\% der Fälle finden [21, 51] (Evidenz III).

- Die fraktionierte Curettage gilt noch als Goldstandard, gibt jedoch nicht in allen Fällen für die zugrundeliegende Erkrankung ein repräsentatives Ergebnis.

Die blinde Dilatation und Curettage kann je nach Autor in bis zu 6\% der Fälle - bei kleinen Zahlen noch häufiger - von Endometriumkarzinom falsch negativ sein [37, 52-55] (Evidenz III u. IV). Die Dilatation und Curettage hat bei gleichem prädikativem Wert bezüglich Endometriumkarzinom eine höhere Komplikationsrate als Pipelle oder Vabra und ist mit bis zu 10fach höheren Kosten verbunden [12, 56].

Zur Abklärung sonographisch sichtbarer Veränderungen unter Tamoxifen genügt die konventionelle Curettage oft nicht, weshalb nach Möglichkeit eine hysteroskopische Endometriumquadrantenbiopsie oder Endometriumresektion vorzuziehen ist [18] (Evidenz IV).

- Der blinden Gewebeentnahme (Biopsie, fraktionierte Curettage) ist die hysteroskopisch gezielte Biopsie bei der primären Abklärung jeder PMPB vorzuziehen, bei rezidivierenden PMPB ist sie notwendig.

Eine höhere Sensitivität und Spezifität als die Sonographie und/oder Aspirationscurettage hat die $\mathrm{Hy}$ steroskopie mit gezielter Endometriumbiopsie unter Praxisbedingungen ("office hysteroscopy"). Sowohl vom Zeitaufwand, welcher im Bereich von 5-10 Minuten [57] liegt, als auch von seiten der Schmerzhaftigkeit her [57], wird diese Methode in der englischsprachigen Literatur vorgezogen [58] (Evidenz III u. IV). Polypen können gleichzeitig entfernt werden. Die Office Hysteroscopy hat ein gutes Kosten-Nutzen-
Verhältnis. Bis zu 60\% der Hospitalisationen wegen PMPB können angeblich eingespart werden $[19,57]$ (Evidenz IV).

Folgende Nachteile des Verfahrens verhinderten allerdings eine weite Etablierung:

1) Bei einer Zervikalstenose kann der Eingriff nicht durchgeführt werden.

2) Die Invasivität schliesst die Gefahr von Perforationen in sich [59].

3) Die vor allem in der Anfangszeit aufgetretenen Komplikationen im Zusammenhang mit der Insufflation von $\mathrm{CO}_{2}$-Gas $[59,60]$.

4) Nach $\mathrm{CO}_{2}$-Insufflation kann es zu postoperativen Schulterschmerzen kommen [61].

5) Die Methode ist an einen infrastrukturellen Mehraufwand gebunden.

Daneben bleibt zu erwähnen, dass die richtige Beurteilung des Cavum uteri, welche zur gezielten Biopsie notwendig ist, ein hohes Mass an Erfahrung voraussetzt.

Eine Umfrage bei sämtlichen niedergelassenen Gynäkologen in Schottland hat gezeigt, dass auch dort die Office Hysteroscopy mit Biopsie nicht etabliert ist und nur 17\% der Befragten sie in der Endometriumabklärung anwenden [62].

In letzter Zeit wird als Distensionsmedium auch in der Praxis zunehmend Flüssigkeit verwendet.

Die Office Hysteroscopy mit Endometriumbiopsie überzeugt vom Aufwand und Ertrag her. Sie erfordert aber eine entsprechende Einrichtung und Erfahrung und hat sich bei uns vorerst nicht durchgesetzt.

Die diagnostische Hysteroskopie in der Tagesklinik ("outpatient hysteroscopy") ist mit der Office Hysteroscopy zu vergleichen. Die Patientin kann am Tag des Eingriffes die Klinik wieder verlassen. Aufgrund der gewonnenen Ergebnisse kann der Arzt eine allenfalls notwendige operative Hysteroskopie oder andere Therapieoptionen mit der Patientin besprechen. Als Distensionsmedium werden $\mathrm{CO}_{2}$-Gas oder $\mathrm{NaCl}$ 0,9\%, seltener 32\% Dextran 70 verwendet. In der Regel kommt ein 4 oder $5 \mathrm{~mm}$ Hysteroskop mit $30^{\circ}$-Optik zum Einsatz [19, 26, 63, 65, 66]. Die Analgesie wird unterschiedlich gehandhabt. Während De Jong [61] generell einen Parazervikalblock setzt, kommt er bei Nagele in einer Serie von 2500 Patientinnen nur bei Bedarf - insbesondere bei Dilatation des Zervikalkanales - zum Einsatz [64]. Wenn die Patientin anästhesiert ist, besteht die Möglichkeit für kleine hysteroskopische Eingriffe [64].

Die diagnostische Hysteroskopie mit Dilatation und Curettage erfordert meistens eine ambulante Spitalaufnahme oder eine Kurzhospitalisation, obschon z.B. Gimpelson [65] die Hysteroskopie und Curettage als "office surgery" durchführt. Die Dilatation macht eine lokale, evtl. eine regionale Analgesie oder eine Vollnarkose notwendig [66]. Neben diesen Nachteilen bietet die Methode nennenswerte Vorteile: Sie eignet sich auch für Patientinnen, deren Allgemeinzustand einen ambulanten Eingriff verunmöglicht. Die Sensitivität und Spezifität liegen noch höher als bei der diagnostischen Hysteroskopie mit gezielter Biopsie, 
denn trotz inspektorisch unauffälligem Endometrium kann sich histologisch ein Endometriumkarzinom finden, welches der gezielten Biopsie entgangen wäre [19, 26, 65, 66] (Evidenz III). Aus diesem Grund gehört in Weiterbildungskliniken wie zum Beispiel Zürich die Curettage mit vorgängiger Hysteroskopie zum Standardvorgehen bei auffälligen sonographischen oder zytologischen Befunden nach PMPB [67]. Auch in Bern, wo in einem Kollektiv von 166 Patientinnen mit PMPB vier Karzinome hysteroskopisch verpasst und erst in der histologischen Aufarbeitung entdeckt wurden, gilt die gleiche Vorgehensweise (präliminäre Daten). Ein zweizeitiges Vorgehen, wie es von Gücer [26] gefordert wird, bringt keinen zusätzlichen Vorteil.

Eine Hysteroskopie als Kontrolle unmittelbar im Anschluss an die Curettage ermöglicht festzustellen, ob allfällige Polypen oder suspekte Befunde auch tatsächlich entfernt wurden, denn bei der blinden Dilatation und Curettage werden oftmals weniger als 75\% des Cavum uteri erfasst [12, 55]. Verbleibende Polypen nach Curettage können mittels Hysteroskopie entfernt werden [68].

Auch Hysteroskopie plus Curettage können keine 100prozentige Sensitivität und Spezifität erreichen. Es stimmen aber alle Autoren darin überein, dass sich die beiden Methoden in ihrer diagnostischen Aussagekraft bei PMPB ergänzen. Hyperplasie und Karzinome werden vor allem bei der Curettage häufiger erfasst, dagegen ist die Hysteroskopie bei der Entdeckung von Polypen und Myomen überlegen $[19,65,66]$.
Die Hysteroskopie mit Endometriumbiopsie oder Curettage sollte als neuer Goldstandard in der Abklärung von PMPB mit auffälligen sonographischen Befunden angesehen werden (Tab. 5 und 6) [69] (Evidenz III).

- Die Hysterektomie ist bei technisch nicht abklärbaren rezidivierenden PMPB als letzte diagnostische Methode indiziert.

Die Dilatation und Curettage galt während mehr als einem Jahrhundert als Goldstandard in der $\mathrm{Ab}$ klärung und zum Teil auch Therapie der PMPB. Bei rezidivierenden Blutungen oder unklaren histologischen Befunden war häufig die Hysterektomie die einzige Lösung. Durch die Entwicklung von Sonographie, diagnostischer und therapeutischer Hysteroskopie kann eine Hysterektomie bei PMPB oft vermieden werden.

Auch bei persistierender PMPB darf bei Vorliegen einer benignen Histologie zugewartet werden, da ein Endometriumkarzinom unwahrscheinlich ist. Regelmässige Nachkontrollen sind aber geboten. Diese Empfehlung ist einer retrospektiven Studie mit nur 40 Hysterektomien entnommen [70].

Kann wegen einer unüberwindbaren Zervikalstenose keine Abklärung ausser der Vaginalsonographie erfolgen, sollte eine Hysterektomie bei auffälligem sonographischem Befund in Betracht gezogen werden [49, 68, 70] (Evidenz III u. IV).

Die Indikation zur Abklärung einer PMPB mittels Hysterektomie ist mit den heutigen diagnostischen Möglichkeiten nur noch in wenigen Fällen gegeben. 


\section{Anhang}

\section{Tabelle 1}

Prävalenz der Endometriumatrophie bei Patientinnen mit PMPB in bezug auf mittels TVS gemessener Endometriumdicke.

\begin{tabular}{|c|c|c|c|c|}
\hline $\begin{array}{c}\text { Anzahl Patientinnen } \\
\text { mit PMPB }\end{array}$ & $\begin{array}{c}\text { Anzahl } \\
\text { Atrophien }\end{array}$ & $\begin{array}{l}\text { korrekte Diagnose } \\
\leq 4 \mathrm{~mm}\end{array}$ & $\begin{array}{l}\text { nicht korrekte Diagnose } \\
>4 \mathrm{~mm}\end{array}$ & Literatur \\
\hline 179 & 106 & 90 & 16 & 29 \\
\hline 205 & 150 & 150 & - & 16 \\
\hline 103 & 33 & 32 & 1 & 25 \\
\hline 179 & 117 & 117 & - & 17 \\
\hline 129 & 63 & 58 & 5 & 32 \\
\hline 1168 & 667 & 491 & 176 & 11 \\
\hline 232 & 59 & 55 & 4 & 34 \\
\hline 195 & 50 & 34 & 16 & 8 \\
\hline 96 & 30 & 28 & 2 & 3 \\
\hline 250 & 162 & 151 & 11 & 5 \\
\hline 930 & 458 & 262 & 196 & 20 \\
\hline 166 & 108 & 93 & 15 & 35 \\
\hline 162 & 75 & 60 & 15 & 9 \\
\hline 259 & 29 & 21 & 8 & 1 \\
\hline 103 & 52 & 52 & - & 26 \\
\hline 149 & 87 & 59 & 28 & 38 \\
\hline 353 & 92 & 69 & 23 & 33 \\
\hline 4858 & 2338 & 1822 & 516 & \\
\hline
\end{tabular}

\section{Tabelle 2}

Prävalenz des Endometriumkarzinoms bei Patientinnen mit PMPB in bezug auf mittels TVS gemessener Endometriumdicke.

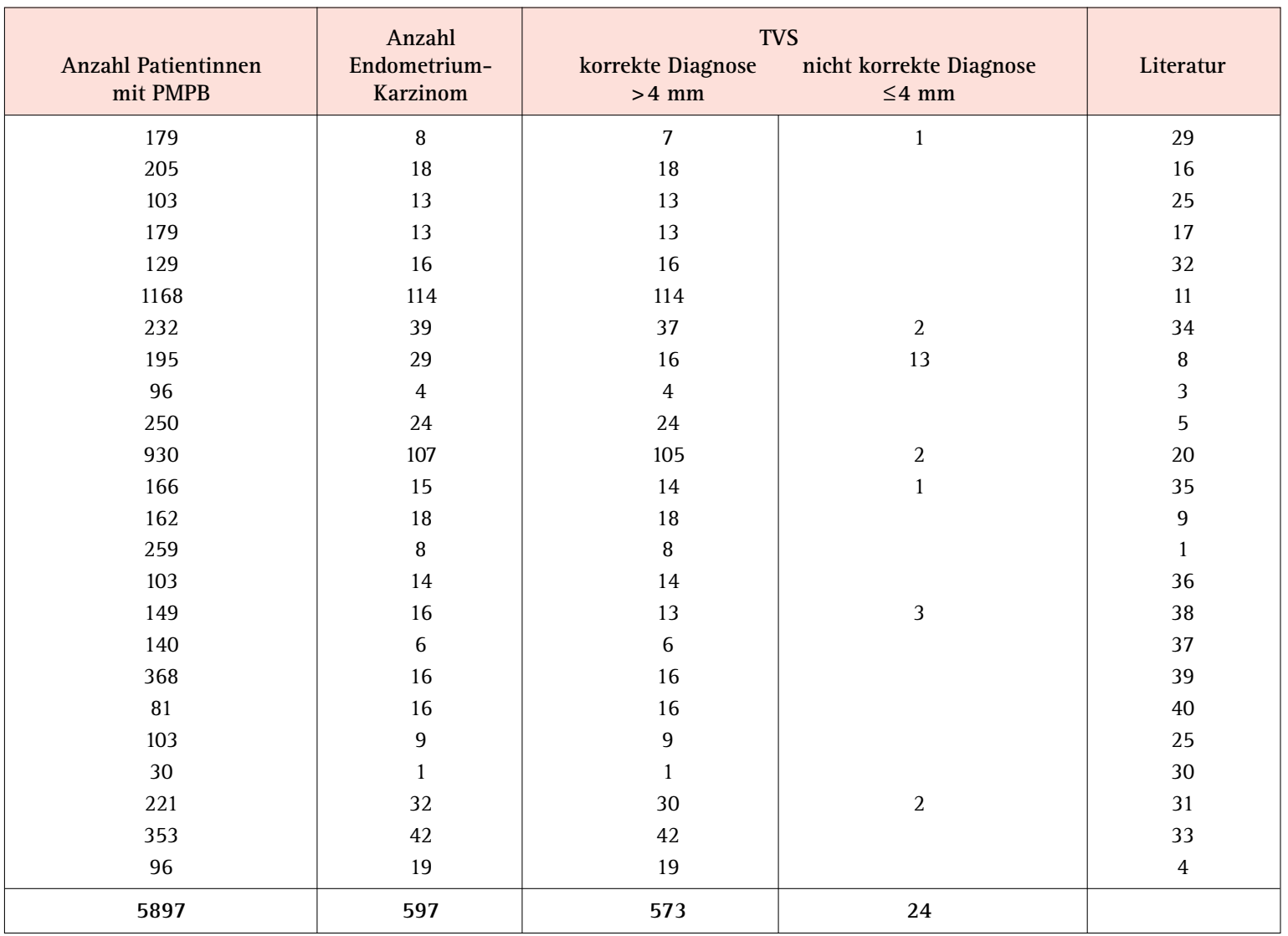




\section{Tabelle 3}

Prävalenz der Endometriumpolypen bei Patientinnen mit PMPB in bezug auf mittels TVS gemessener Endometriumdicke.

\begin{tabular}{|c|c|c|c|c|}
\hline 179 & 25 & 25 & & 17 \\
\hline 129 & 14 & 14 & & 32 \\
\hline 232 & 38 & 25 & 13 & 34 \\
\hline 96 & 7 & 6 & 1 & 3 \\
\hline 250 & 19 & 19 & & 5 \\
\hline 930 & 97 & 84 & 13 & 20 \\
\hline 166 & 5 & 5 & & 35 \\
\hline 96 & 15 & 13 & 2 & 4 \\
\hline 353 & 37 & 27 & 10 & 33 \\
\hline 4404 & 492 & 437 & 55 & \\
\hline
\end{tabular}

\section{Tabelle 4}

Prävalenz der Endometriumhyperplasien bei Patientinnen mit PMPB in bezug auf mittels TVS gemessener Endometriumdicke.

\begin{tabular}{|c|c|c|c|c|}
\hline $\begin{array}{c}\text { Anzahl Patientinnen } \\
\text { mit PMPB }\end{array}$ & $\begin{array}{c}\text { Anzahl } \\
\text { Hyperplasien }\end{array}$ & $\begin{array}{l}\text { korrekte Diagnose } \\
>4 \mathrm{~mm}\end{array}$ & $\begin{array}{l}\text { nicht korrekte Diagnose } \\
\leq 4 \mathrm{~mm}\end{array}$ & Literatur \\
\hline 179 & 13 & 11 & 2 & 29 \\
\hline 205 & 13 & 13 & & 16 \\
\hline 179 & 13 & 13 & & 17 \\
\hline 129 & 16 & 16 & & 32 \\
\hline 1168 & 112 & 106 & 6 & 11 \\
\hline 232 & 23 & 22 & 1 & 34 \\
\hline 96 & 8 & 8 & & 3 \\
\hline 250 & 45 & 45 & & 5 \\
\hline 930 & 120 & 117 & 3 & 20 \\
\hline 166 & 12 & 12 & & 35 \\
\hline 162 & 19 & 19 & & 9 \\
\hline 259 & 7 & 7 & & 1 \\
\hline 103 & 37 & 37 & & 26 \\
\hline 30 & 4 & 3 & 1 & 30 \\
\hline 96 & 33 & 28 & 5 & 4 \\
\hline 195 & 74 & 50 & 24 & 8 \\
\hline 353 & 50 & 43 & 7 & 33 \\
\hline 4732 & 599 & 550 & 49 & \\
\hline
\end{tabular}




\section{Tabelle 5}

Sensitivität und Spezifität ohne Trennung in prämenopausal und gemischt.

\begin{tabular}{|c|c|c|c|c|c|c|}
\hline \multirow[t]{2}{*}{ Anzahl } & \multicolumn{2}{|c|}{ HSC } & \multicolumn{2}{|c|}{ TVS } & Curettage & \multirow[t]{2}{*}{ Literatur } \\
\hline & Sensitivität & Spezifität & Sensitivität & Spezifität & Sensitivität Spezifität & \\
\hline 229 & 96 & 92,9 & & & & 71 \\
\hline 68 & 97 & 93 & 100 & 80 & & 75 \\
\hline 68 & 78 & 54 & 95 & 65 & & 76 \\
\hline 149 & 79 & 93 & 54 & 90 & & 78 \\
\hline 803 & 71,8 & 96,4 & & & & 79 \\
\hline 1317 & & & & & & \\
\hline
\end{tabular}

\section{Tabelle 6}

Sensitivität und Spezifität postmenopausal.

\begin{tabular}{|c|c|c|c|c|c|c|c|}
\hline Anzahl & \multicolumn{2}{|c|}{ HSC } & \multicolumn{2}{|c|}{ TVS } & \multicolumn{2}{|c|}{ Curettage } & Literatur \\
\hline 103 & 89 & 98 & 100 & 30,8 & & & 26 \\
\hline 39 & 93,7 & 76,9 & & & & & 72 \\
\hline 45 & 86,9 & 91,7 & 73,9 & 95,7 & 100 & 100 & 73 \\
\hline 81 & 95,3 & 93,9 & 95,8 & 45,5 & & & 40 \\
\hline 28 & 100 & 89,4 & 100 & 60,8 & & & 77 \\
\hline 595 & 85,1 & 100 & & & & & 79 \\
\hline 942 & & & & & & & \\
\hline
\end{tabular}

\section{Literatur}

1 Dubinsky TJ, Parvey HR, Maklad N. The role of transvaginal and endometrial biopsy in the evaluation of peri- and postmenopausal bleeding. Am J Roentgenol 1997;169:145-9.

2 Nagele F, O'Connor H, Baskett TF, Davies A, Mohammed H, et al. Hysteroscopy in women with abnormal uterine bleeding on hormone replacement therapy: a comparison with postmenopausal bleeding. Fertil Steril 1996;65(6):1145-50.

3 Wolman I, Sagi J, Ginat S, Jaffa AJ, Hartoov J, Jedwab G. The sensitivity and specificity of vaginal sonography in detecting endometrial abnormalities in women with postmenopausal bleeding. J Clin Ultrasound 1996;24:79-82.

4 Pertl B, Lahousen M, Pieber D, Heidarfadai HJ, Giuliani A. Stellenwert der Sonographie bei der Früherkennung des Endometriumkarzinoms. Gynäkol Geburtshilfliche Rundsch 1996;36:14-20

5 Grigoriou 0, Kalovidouros A, Papadias C, Antoniou G, Antonaki V, Giannikos L. Transvaginal sonography of the endometrium in women with postmenopausal bleeding. Maturitas 1996;23:9-14

6 Novak's GYNECOLOGY. 12 th edition. Baltimore: Williams Wilkings; 1996. p. 1057-110.

7 Lee WH, Tan KH, Lee YW. The aetiology of postmenopausal bleeding - a study of 163 consecutive cases in Singapore. Singapore Med J 1995;36:164-8.

8 Schramm T, Kürzl R, Schweighart C, Stuckert-Klein AC. Endometriumkarzinom und Vaginalsonographie: Untersuchungen zur diagnostischen Validität. Geburtshilfe und Frauenheilkunde 1995;55:65-72.

9 Mateos F, Zarauz R, Seco C, Rayward JR, del Barrio P, Aguirre J, Bajo JM. Assessment with transvaginal ultrasonography of endometrial thickness in women with postmenopausal bleeding. Eur J Gynecol Oncol 1997;18:504-7.

10 Timmerman D, Vergote J. Endometrial cancer and uterine sarcoma. In: Brosens J, Wamsteker K. Diagnostic imaging and endoscopy in gynecology. A practical guide. London: W. B. Saunders; 1997. p. 199-212.
11 Karlsson B, Granberg S, Wikland M, Yostalo P, Marsal K, Valentin L. Transvaginal ultrasonograpy of the endometrium in women with postmenopausal bleeding - a Nordic multicenter study. Am J Obstet Gynecol 1995;172:1488-94.

12 Grimes DA. Diagnostic dilatation and curettage: a reappraisal. Am J Obstet Gynecol 1982;142:1-6.

13 Gredmark T, Kvint S, Havel G, Mattsson LA. Histopathological findings in women with postmenopausal bleeding. Br J Obstet Gynecol 1995;102:133-6.

14 Questions and answers on breast cancer. A guide for women and their physicians. Steering Committee on Clinical Practice Guidelines for the Care and Treatment of Breast Cancer. CMAJ 1998;158(3 suppl):1-31.

15 Keller PJ. Grundlagen und Definitionen. In: Keller PJ. Menopause. Bern: Bäbler-Verlag; 1995. S. 7-11.

16 Granberg S, Wikland M, Karlsson B, Norström A, Fiberg L. Endometrial thickness as measured by endovaginal ultrasonography for identifying endometrial abnormality. Am J Obstet Gynecol 1991;164:47-52.

17 Nasri MN, Shepherd JH, Setchell ME, Lowe DG, Chard T. Sonographic depiction of postmenopausal endometrium with transabdominal and transvaginal scanning. Ultrasound Obstet Gynecol 1991;1:279-83.

18 Rageth JC, Bronz L, Granberg S. Tamoxifen und Endometrium: Überwachung und Abklärung endometrialer Veränderungen. Arch Gynecol Obstet 1995;256 (Suppl):122-7.

19 Gupta JK, Wilson S, Desai P, Hau C. How should we investigate women with postmenopausal bleeding? Acta Obstet Gynecol Scand 1996;75:475-9.

20 Ferrazzi E, Torri V, Trio D, Zannoni E, Filiberto S, Dordoni D. Sonographic endometrial thickness: a useful test to predict atrophy in patients with postmenopausal bleeding. An Italian multicenter study. Ultrasound Obstet Gynecol 1996;7:315-21.

21 Townsend DE, Fields G, McCausland A, Kauffman K. Diagnostic and operative hysteroscopy in the management of persistent postmenopausal bleeding. Obstet Gynecol 1993;82:419-21. 
22 Cohen JR, Luxman D, Sagi J, Yovel I, Wolman I, David MP. Sonohysterography for distinguishing endometrial thickening from endometrial polyps in postmenopausal bleeding. Ultrasound Obstet Gynecol 1994;4:227-30.

23 Cravello L, de Montgolfier R, D’Ercole C, Boubli L, Blanc B. Hysteroscopic surgery in postmenopausal women. Acta Obstet Gynecol Scand 1996;75:563-6.

24 Bronz L, Suter T, Rusca T. The value of transvaginal sonography with and without saline instillation in the diagnosis of uterine pathology in pre- and postmenopausal women with abnormal bleeding or suspect sonographic findings. Ultrasound Obstet Gynecol 1997;9:53-8.

25 Osmers R, Völksen M, Schauer A. Vaginosonography for early detection of endometrial carcinoma. Lancet 1990; 335:1569-71.

26 Gücer F, Arikan MG, Petru E, Mitterdorfer B, Lahousen M, Lax S. Aussagekraft der kombinierten Anwendung von Vaginalsonographie und Hysteroskopie bei peri- und postmenopausalen Blutungen. Gynäkol Geburtshilfliche Rundsch 1996;36:9-13.

27 Volgger B, Alge A, Windbichler G, Zeimet AG, MüllerHolzner E, Marth C. Sonomorphologische Beurteilung des postmenopausalen Endometriums. Eine prospektive Studie. Gynäkol Geburtshilfliche Rundsch 1996;36:21-8.

28 Haller U. Maligne Tumoren des Corpus uteri. In Bender HG, Diedrich K, Künzel W. Allgemeine Gynäkologische Onkologie. München: Urban und Schwarzenberg; 1998. S. 191.

29 Klug PW, Leitner G. Die Gegenüberstellung vaginalsonographischer und histologischer Befunde am Endometrium. Geburtshilfe und Frauenheilkunde 1989;49:797-802.

30 Goldstein SR, Nachtigall M, Snyder JR, Nachtigall L. Endometrial assessment by vaginal ultrasonography before endometrial sampling in patients with postmenopausal bleeding. Am J Obstet Gynecol 1990;163:119-23.

31 Brandner P, Gnirs J, Neis KJ, Hettenbach A, Schmidt W. Der Stellenwert der Vaginosonographie in der non-invasiven Beurteilung des Endometriums am postmenopausalen Uterus. Geburtshilfe und Frauenheilkunde 1991;51:734-40.

32 Auslender R, Bornstein J, Dirnfeld M, Kogan 0, Atad J, Abramovici H. Vaginal ultrasonography in patients with postmenopausal bleeding. Ultrasound Obstet Gynecol 1993; 3:426-28.

33 Taipale P, Tarjanne H, Heinonen UM. The diagnostic value of transvaginal sonography in the diagnosis of endometrial malignancy in women with peri- and postmenopausal bleeding. Acta Obstet Gynecol Scand 1994;73:819-23.

34 Seelbach-Göbel B, Rempen A, Kristen P. Transvaginaler Ultraschall am Endometrium in der Postmenopause. Geburtshilfe und Frauenheilkunde 1995;55:59-64.

35 Tsuda H, Kawabata M, Yamamoto K, Inoue T, Umesaki N. Prospective study to compare endometrial cytology and transvaginal ultrasonography for identification of endometrial malignancies. Gynecol Oncol 1997;65:383-6.

36 Fistonic I, Hodek B, Klaric P, Jokanovic L, Grubisic G, Ivicevic-Bakulic T. Transvaginal sonographic assessment of premalignant and malignant changes in the endometrium in postmenopausal bleeding. J Clin Ultrasound 1997;25:431-5.

37 Van den Bosch T, Vandendael A, Van Schoubroeck D, Wranz PA, Lombard CJ. Combining vaginal ultrasonography and office endometrial sampling in the diagnosis of endometrial disease in postmenopausal women. Obstet Gynecol 1995;85:349-52.

38 Conoscenti G, Meir YJ, Fischer Tamaro L, Maieron A, Natale R, D'Ottavio G, et al. Endometrial assessment by transvaginal sonography and histological findings after D\&C in women with postmenopausal bleeding. Ultrasound Obstet Gynecol 1995;6:108-15.

39 Cecchini S, Ciatto S, Bonardi R, Grazzini G, Mazzotta A. Endometrial ultrasonography - An alternative to invasive assessment in women with postmenopausal vaginal bleeding. Tumori 1996;82:38-9.

40 Haller H, Matecjcic N, Rukavina B, Krasevic M, Rupcic S, Mozetic D. Transvaginal sonography and hysteroscopy in women with postmenopausal bleeding. Int J Gynecol Obstet 1996;54:155-9.
41 De Blok S. Infections, atrophy, hyperplasia and polyps of the endometrium. In: Brosens J, Wamsteker K. Diagnostic imaging and endoscopy in gynecology. A practical guide. London: W. B. Saunders; 1997. p. 147-61.

42 Weigel M, Friese K, Strittmatter HJ, Melchert F. Measuring the thickness - is that all we have to do for sonographic assessment of endometrium in postmenopausal women? Ultrasound Obstet Gynecol 1995;6:97-102.

43 Karlsson B, Granberg S, Ridell B, Wikland M. Endometrial thickness as measured by transvaginal sonography: interobserver variation. Ultrasound Obstet Gynecol 1994;4:320-5.

44 Dubinsky TJ, Parvey HR, Gormaz G, Curtis M, Maklad N. Transvaginal hysterosonography: comparison with biopsy in the evaluation of postmenopausal bleeding. J Ultrasound Med 1995;14:887-93.

45 Rudigoz RC, Salle B, Piacenza JM, de Saint-Hilaire P, Gaucherand P. Etude de la cavité utérine par hystérosonographie. J Gynecol Obstet Biol Reprod 1995;24:697-704.

46 Bernard JP, Lecuru F, Darles C, Robin F, De Bièvre P, Taurelle R. Utilisation de l'échographie avec accentuation de contraste comme examen de première intention dans l'exploration de la cavité utérine. J Gynecol Obstet Biol Reprod 1998;27:167-73.

47 Schwärzler P, Concin H, Bösch H, Berlinger A, Wohlgenannt K, Collins WP, Bourne TH. An evaluation of sonohysterography and diagnostic hysteroscopy for the assessment of intrauterine pathology. Ultrasound Obstet Gynecol 1998;11:337-42.

48 O'Connell LP, Fries MH, Zeringue E, Brehm W. Triage of abnormal postmenopausal bleeding: A comparison of endometrial biopsy and transvaginal sonohysterography versus fractional curettage with hysteroscopy. Am J Obstet Gynecol 1998;178:956-61.

49 Brooks PG, Serden SP. Hysteroscopic findings after unsuccessful dilatation and curettage for abnormal uterine bleeding. Am J Obstet Gynecol 1988;158:1354-7.

50 Emanuel MH, Verdel MJ, Wamstecker K, Lammes FB. A prospective comparison of transvaginal ultrasonography and diagnostic hysteroscopy in the evaluation of patients with abnormal uterine bleeding: clinical implications. Am J Obstet Gynecol 1995;172:547-52.

51 Chambers JT, Chambers SK. Endometrial Sampling: when? where? why? with what? Clin Obstet Gynecol 1992;35:28-39.

52 Goldschmit R, Katz Z, Blickstein I, Caspi B, Dgani R. The accuracy of endometrial pipelle sampling with and without sonographic measurement of endometrial thickness. Obstet Gynecol 1993;82:727-30.

53 Stovall TG, Solomon SK, Ling FW. Endometrial sampling prior to hysterectomy. Obstet Gynecol 1989;73:405-9.

54 Krampl E, Soby B, Istre 0. How representative are Pipelle endometrial biopsies? A retrospective analysis of 324 biopsies followed by transcervical resection of the endometrium or hysterectomy. Gynecol Endosc 1997;6:277-81.

55 Lerner HM. Lack of efficacy of prehysterectomy curettage as a diagnostic procedure. Am J Obstet Gynecol 1984; 148:1055-6.

56 Feldman S, Berkowitz RS, Tosteson ANA. Cost-effectiveness of strategies to evaluate postmenopausal bleeding. Obstet Gynecol 1993;81:968-75.

57 Bradley LD, Widrich T. State-of-the-art flexible hysteroscopy for office gynecologic evaluation. J Am Assoc Gynecol Laparosc 1995;2(3):263-7.

58 Downes E, Al-Azzaw F. The predictive value of outpatient hysteroscopy in a menopause clinic. Br J Obstet Gynaecol 1993;100:1148-9.

59 De Bruyne F, Somville T, Hucke J. Komplikationen bei der Hysteroskopie. Gynäkologe 1993;26:385-8.

60 Loffer FD. Complications from uterine distention during hysteroscopy. In: Corfman RS, Diamond MP, De Cherney A (eds.). Complications of laparoscopy and hysteroscopy. Oxford: Blackwell Science; 1997. p. 177-86.

61 De Jong P, Doel F, Falconer A. Outpatient diagnostic hysteroscopy. Br J Obstet Gynaecol 1990;97:299-303.

62 Penney G, Vale L, Souter V, Templeton A. Endometrial assessment procedures: an audit of current practice in Scotland. Hum Reprod 1997;12(9):2041-5. 
63 Deix R. Stellenwert der Hysteroskopie in der modernen Gynäkologie. Gynäkol Geburtshilfliche Rundsch 1995; 35:116-8.

64 Nagele F, 0'Connor H, Davies A, Badawy A, Mohamed H, Magos A. 2500 outpatient diagnostic hysteroscopies. Obstet Gynecol 1996;88:87-92.

65 Gimpelson RJ, Rappold H0. A comparative study between panoramic hysteroscopy with directed biopsy and dilatation and curettage. Am J Obstet Gynecol 1988;158:489-92.

66 Ben-Yehuda OM, Kim YB, Leuchter RS. Does hysteroscopy improve upon the sensitivity of dilatation and curettage in the diagnosis of endometrial hyperplasia or carcinoma? Gynecol Oncol 1998;68:4-7.

67 Köchli OR, Schär GN, Bajka M, Pok Lundquist J, Nussbaumer R, Keller PJ, Haller U. Analyse der Indikationen und Resultate der fraktionierten Curettage an einem grossen gynäkologischen Krankengut. Schweiz Med Wochenschr 1996;126(3):69-76.

68 Concin H, Bösch H, Schwärzler P. Hysteroskopie-Stellenwert und Risiko. Gynäkol Geburtshilfliche Rundsch 1995;35:114-9.

69 Mortakis AE, Mavrelos K. Transvaginal ultrasonography and hysteroscopy in the diagnosis of endometrial abnormalities. J Am Assoc Gynecol Laparosc 1997;4:449-52.

70 Fung Kee Fung M, Burnett M, Faught W. Does persistent postmenopausal bleeding justify hysterectomy? Eur J Gynaecol Oncol 1997;18(1):26-8.

71 Valli E, Zupi E, Montevecchi L, Marconi D, Dini ML, et al. A new hysteroscopic classification of and nomenclature for endometrial lesions. J Am Assoc Gynecol Laparosc 1995; 2:279-83.
72 Altaras MM, Aviram R, Cohen I, Markov S, Goldberg GL, et al. Microhysteroscopy and endometrial sampling biopsy results following failed diagnostic dilatation and curettage in women with postmenopausal bleeding. Int J Gynecol Obstet 1993;42:255-60.

73 Cacciatore B, Ramsay T, Lehtovirta P, Ylstalo P. Transvaginal sonography and hysteroscopy in postmenopausal bleeding. Acta Obstet Gynecol Scand 1994;73:413-6.

74 Karlsson B, Granberg S, Hellberg P, Wikland M. Comparative study of transvaginal sonography and hysteroscopy for the detection of pathologic endometrial lesions in women with postmenopausal bleeding. J Ultrasound Med 1994;13:757-62.

75 Widrich T, Bradley LD, Mitchinson AR, Collins RL. Comparison of saline infusion sonography with office hysteroscopy for the evaluation of the endometrium. Am J Obstet Gynecol 1996;174:1327-34

76 Saidi MH, Sadler RK, Theis VD, Akright BD, Farhart SA, et al. Comparison of sonography, sonohysterography and hysteroscopy for evaluation of abnormal uterine bleeding. J Ultrasound Med 1997;16(9):587-91.

77 Alcazar JL, Laparte C. Comparative Study of transvaginal ultrasonography and hysteroscopy in postmenopausal bleeding. Gynecol Obstet Invest 1996;41:47-9.

78 Townbin N, Gviazda IM, March CM. Office hysteroscopy versus transvaginal ultrasonography in the evaluation of patients with excessive uterine bleeding. Am J Obstet Gynecol 1996;174:1678-82.

79 Torrejon R, Fernandez-Alba JJ, Carnicer I, Martin A, Castro C, et al. The value of hysteroscopic exploration for abnormal uterine bleeding. J Am Assoc Gynecol Laparosc 1997;4:453-6.

La version française suivra 